\title{
A Study on Differential Private Online Learning
}

\author{
Weilin Nie, Cheng Wang \\ Huizhou University, Huizhou, China \\ Email: niewl@hzu.edu.cn,wangch@hzu.edu.cn
}

How to cite this paper: Nie, W.L. and Wang, C. (2017) A Study on Differential Private Online Learning. Journal of Computer and Communications, 5, 28-33. https://doi.org/10.4236/jcc.2017.52004

Received: December 18, 2016

Accepted: February 18, 2017

Published: February 21, 2017

Copyright $\odot 2017$ by authors and Scientific Research Publishing Inc. This work is licensed under the Creative Commons Attribution International License (CC BY 4.0).

http://creativecommons.org/licenses/by/4.0/

\begin{abstract}
Online learning algorithms are very attractive, in which iterations are applied efficiently instead of solving some optimization problems. In this paper, online learning with protecting privacy is considered. A perturbation term is added into the classical online algorithms to obtain the differential privacy property. Firstly the distribution for the perturbation term is deduced, and then an error analysis for the new algorithms is performed, which shows the convergence and learning rate. From the error analysis, a choice for the parameters for differential privacy can be found theoretically.
\end{abstract}

\section{Keywords}

Online Learning, Differential Privacy, Output Perturbation, Error

Decomposition, Learning Rate

\section{Introduction}

Online learning is widely used recently in computer sciences, due to its efficiency in calculation and well theoretical results. Compared with the classical batch learning in learning theory, online algorithms update the output only according to the last sample point. So such algorithms are very effective to handle the practical problems and have been studied in [1] [2] [3] [4] [5] and etc. However, as the technologic development of data analysis, there are risks for applying such algorithms on a big data set. A commonly used notion for measuring the risk is differential privacy [6]. Little references on this topic can be found except for [7]. There the authors conducted an analysis for online convex programming. Choice for the parameters of differential privacy and utilities analysis are presented for algorithms such as implicit gradient descent and generalized infinitesimal gradient descent. In this paper, the line of work begins with [8] is considered which can be thought as a kernel online learning algorithm. 


\section{Fundamental Principles}

Our setting for online learning is introduced as follow. Let the input space $X$ be a compact metric space, and output $Y \in[-M, M]$ for some $M>0$ as a regression problem. Denote $Z:=X \times Y$ as the sample space. Assume there is a probability measure $\rho$ on $Z$, which can be decomposed to marginal distribution $\rho_{X}$ on $X$ and conditional distribution $\rho(y \mid x)$ on $Y$ at $x \in X$. Then the regression function is defined by

$$
f_{\rho}=\int_{Y} y \mathrm{~d} \rho(y \mid x)
$$

which is indeed the conditional expectation of $y$ given $x$. The regression function minimizes the least square generalization error (see [9] for more details)

$$
\mathcal{E}(f):=\int_{Z}(f(x)-y)^{2} \mathrm{~d} \rho
$$

So learning algorithms always aim to approximate the regression function based on samples $\left\{z_{t}=\left(x_{t}, y_{t}\right)\right\}_{t=0,1,2, \cdots}$, which are drawn independently from distribution $\rho$. Let $K: X \times X \rightarrow R$ be a Mercer Kernel, and $\mathcal{H}_{K}$ is the induced reproducing kernel Hilbert space (RKHS, [10]), i.e., the completion of $\operatorname{span}\left\{K_{x}: x \in X\right\}$ where $K_{x}\left(x^{\prime}\right)=K\left(x, x^{\prime}\right)$ for any $x, x^{\prime} \in X$ with respect to the inner product $\left\langle K_{x}, K_{x^{\prime}}\right\rangle_{K}=K\left(x, x^{\prime}\right)$. The corresponding norm in $\mathcal{H}_{K}$ is denoted as $\|\cdot\|_{K}$. Now our online learning algorithm as

$$
f_{t+1}=f_{t}-\eta_{t}\left[\left(f_{t}\left(x_{t}\right)-y_{t}\right) K_{x_{t}}+\lambda_{t} f_{t}\right], \quad t=0,1,2, \cdots
$$

with $f_{0}=0$. Here $\eta_{t}>0$ is the step size and $\lambda_{t}>0$ is the regularization parameter.

When applying this online algorithm on private data set, it may leak some sensitive information. To deal with this privacy problem, Dwork et al. introduced differential privacy in [11]. Which can be described as follow. For the sample space $Z$ introduced above, the Hamming distance between two sample sets $\left\{z_{1}, z_{2}\right\} \in Z^{m}$ is

$$
\mathrm{d}\left(z_{1}, z_{2}\right)=\#\left\{i=1, \cdots, m: z_{1, i} \neq z_{2, i}\right\}
$$

Definition $1 A$ random algorithm $A: Z^{m} \rightarrow \operatorname{Range}(A)$ is $\epsilon$-differential private if for every two data sets $z_{1}, z_{2}$ satisfying $\mathrm{d}\left(z_{1}, z_{2}\right)=1$, and every set $\mathcal{O} \in \operatorname{Range}\left(A\left(z_{1}\right)\right) \cap \operatorname{Range}\left(A\left(z_{2}\right)\right)$, there holds

$$
\operatorname{Pr}\left\{A\left(z_{1}\right) \in \mathcal{O}\right\} \leq e^{\epsilon} \cdot \operatorname{Pr}\left\{A\left(z_{2}\right) \in \mathcal{O}\right\}
$$

To endow our online algorithm the differential privacy property, a perturbation term is added into the output of (3), that is,

$$
f_{t, \mathcal{A}}=f_{t}+b_{t}
$$

where $b_{t}$ takes value in $R$ with distribution to be determined in following analysis.

Differential private online learning has already been studied in [7], there the authors consider a differentially private online convex programming problem. 
Here our algorithm is different, which is based on the Mercer kernels. Our purpose in this paper is to firstly provide the explicit density function for $b$ and then conduct an error analysis for (6), which reveals the learning rate.

\section{Differential Privacy Analysis}

In this section, a detail analysis for the perturbation term $b_{t}$ in algorithm (6) will be conducted. Firstly recall the useful definition of sensitivity and lemma proposed in [11].

Definition 2 denote $\Delta f_{t}$ as the maximum infinite norm of difference between the outputs when changing the last sample point in $z$. Let $z=\left\{\left(x_{i}, y_{i}\right)\right\}_{i=0}^{t}$ and $z=\left\{\left(x_{1}, y_{1}\right),\left(x_{2}, y_{2}\right), \cdots,\left(x_{t-1}, y_{t-1}\right),\left(x_{\bar{t}}, y_{\bar{t}}\right)\right\}, f_{t}$ and $f_{\bar{t}}$ derived from (3) accordingly, it is clear that

$$
\Delta f_{t}:=\sup _{z, \bar{z}}\left\|f_{t}-f_{\bar{t}}\right\|_{\infty}
$$

Then a similar result to [11] is:

Lemma 1 Assume $\Delta f_{t}$ is bounded by $C_{t}>0$, and $b_{t}$ has density function proportion to $\exp \left\{-\frac{\epsilon|b|}{C_{t}}\right\}$, then algorithm (6) provides $\epsilon$-differential privacy.

Proof. For all possible output function $r$, and $z, \bar{z}$ differ in last element, then

$$
\operatorname{Pr}\left\{f_{t, \mathcal{A}}=r\right\}=\operatorname{Pr}_{b_{t}}\left\{b_{t}=r-f_{t}\right\} \propto \exp \left(-\frac{\epsilon\left|r-f_{t}\right|}{C_{t}}\right)
$$

and

$$
\operatorname{Pr}\left\{f_{\bar{t}, \mathcal{A}}=r\right\}=\operatorname{Pr}_{b_{t}}\left\{b_{t}=r-f_{\bar{t}}\right\} \propto \exp \left(-\frac{\epsilon\left|r-f_{\bar{t}}\right|}{C_{t}}\right)
$$

So by triangle inequality,

$$
\operatorname{Pr}\left\{f_{t, \mathcal{A}}=r\right\} \leq \operatorname{Pr}\left\{f_{\bar{t}, \mathcal{A}}=r\right\} \times e^{\frac{\epsilon\left|f_{t}-f_{\bar{t}}\right|}{C_{t}}} \leq e^{\epsilon} \operatorname{Pr}\left\{f_{\bar{t}, \mathcal{A}}=r\right\}
$$

Then the lemma is proved by a union bound.

It is obvious that if finding the upper bound for $\Delta f_{t}$, the distribution for $b_{t}$ can be derived. Set $\eta_{t}=1 /\left(t+t_{0}\right)^{\theta}$ and $\lambda_{t}=1 /\left(t+t_{0}\right)^{1-\theta}$ for some $t_{0}>0$ and $0<\theta<1$. Moreover, denote $\kappa=\sup _{x, x^{\prime} \in X} K\left(x, x^{\prime}\right)$ (as $K$ is Mercer Kernel on compact metric space $X)$. The next lemma is taken from [1] to bound $\left\|f_{t}\right\|_{K}$.

Lemma 2 If $t_{0}^{\theta} \geq \kappa^{2}+1$, then for all $t \in \mathbb{N}$, there holds

$$
\left\|f_{t}\right\|_{K} \leq \frac{\kappa M}{\lambda_{t}}
$$

Now the main result for differential privacy for algorithm (6) follows.

Theorem 1 When choosing $\eta_{t}=1 /\left(t+t_{0}\right)^{\theta}$ and $\lambda_{t}=1 /\left(t+t_{0}\right)^{1-\theta}$ for some $\frac{1}{2}<\theta<1$ and $t_{0}^{\theta} \geq \kappa^{2}+1$, let the density function of $b_{t}$ is $\frac{1}{\alpha} \exp \left\{\frac{\epsilon|b|}{C_{t}}\right\}$ with $\alpha=2 C_{t} / \epsilon$ and 


$$
C_{t}=\frac{2\left(\kappa^{2}+1\right) \kappa M}{\left(t-1+t_{0}\right)^{2 \theta-1}}
$$

then the algorithm (6) provides $\epsilon$-differential privacy.

Proof. From (3) there holds

$$
f_{t}=f_{t-1}-\eta_{t-1}\left[\left(f_{t-1}\left(x_{t-1}\right)-y_{t-1}\right) K_{x_{t-1}}+\lambda_{t-1} f_{t-1}\right]
$$

and

$$
f_{\bar{t}}=f_{t-1}-\eta_{t-1}\left[\left(f_{t-1}\left(x_{\bar{t}-1}\right)-y_{\bar{t}-1}\right) K_{x_{\bar{t}-1}}+\lambda_{t-1} f_{t-1}\right]
$$

Then

$$
f_{t}-f_{\bar{t}}=\eta_{t-1}\left[\left(f_{t-1}\left(x_{\bar{t}-1}\right)-y_{\bar{t}-1}\right) K_{x_{\bar{t}-1}}-\left(f_{t-1}\left(x_{t-1}\right)-y_{t-1}\right) K_{x_{t-1}}\right]
$$

From the above lemma $\left\|f_{t-1}\right\|_{K} \leq \frac{\kappa M}{\lambda_{t-1}}$ for all $t$. By the reproducing property that $f(x)=\left\langle f, K_{x}\right\rangle_{K} \leq\|f\|_{K}\left\|K_{x}\right\|_{K} \leq \kappa\|f\|_{K} \quad$ (see [9]),

$$
\left\|f_{t}-f_{\bar{t}}\right\|_{K} \leq 2 \eta_{t-1}\left(\frac{\kappa^{2} M}{\lambda_{t-1}}+M\right) \kappa
$$

Therefore

$$
\Delta f_{t}=\sup _{z, \bar{z}}\left\|f_{t}-f_{\bar{t}}\right\|_{\infty} \leq \frac{2 \kappa^{2}\left(\kappa^{2}+1\right) M}{\left(t-1+t_{0}\right)^{2 \theta-1}}
$$

Set $B_{t}$ to be the right hand side in lemma 1 then the theorem is proved.

\section{Error Analysis}

In this section, $f_{\rho} \in \mathcal{H}_{K}$ is assumed for simplicity. It will be shown that $f_{t}$ obtained from (6) still converge to regression function $f_{\rho}$ by choosing appropriate parameter $\epsilon$ under the choice of $\eta_{t}$ and $\lambda_{t}$ as in the theorem in the last section. To this end, an error decomposition is needed. Denote operators $L_{t}: \mathcal{H}_{K} \rightarrow \mathcal{H}_{K}$ as $L_{t}(f)=f\left(x_{t}\right) K_{x_{t}}$ for $t=0,1,2, \cdots$, and $I$ as the identity operator. It is easy to verify that $\left\|L_{t}\right\| \leq \kappa^{2}$. Notice that $f_{\rho} \in \mathcal{H}_{K}$, the following decomposition can be deduced:

$$
\begin{aligned}
f_{t+1}-f_{\rho} & =\left(I-\eta_{t} \lambda_{t} I-\eta_{t} L_{t}\right)\left(f_{t}-f_{\rho}\right)+\eta_{t}\left[y_{t} K_{x_{t}}-\left(\lambda_{t} I+L_{t}\right) f_{\rho}\right] \\
& =A_{t}\left(f_{t}-f_{\rho}\right)+B_{t}=A_{t}\left[A_{t-1}\left(f_{t-1}-f_{\rho}\right)+B_{t-1}\right]+B_{t}=\cdots \\
= & A_{t} A_{t-1} \cdots A_{0}\left(f_{0}-f_{\rho}\right)+\left[B_{t}+A_{t} B_{t-1}+\cdots+A_{t} A_{t-1} \cdots A_{1} B_{0}\right]
\end{aligned}
$$

Here $A_{t}=I-\eta_{t} \lambda_{t} I-\eta_{t} L_{t}$ and $B_{t}=\eta_{t}\left[y_{t} K_{x_{t}}-\left(\lambda_{t} I+L_{t}\right) f_{\rho}\right]$. In the following the first term is called initial error and second one is sample error. The initial error is easy to bound from the analysis above. Since $t_{0}$ is such that $t_{0}^{\theta} \geq \kappa^{2}+1, A_{t}$ is a positive operator with $\left\|A_{t}\right\| \leq 1-\eta_{t} \lambda_{t}=\left(t+t_{0}-1\right) /\left(t+t_{0}\right)$.

$$
\begin{aligned}
\left\|A_{t} A_{t-1} \cdots A_{0}\left(f_{0}-f_{\rho}\right)\right\|_{K} & \leq\left\|A_{t}\right\|\left\|A_{t-1}\right\| \cdots\left\|A_{0}\right\| \cdot\left\|f_{0}-f_{\rho}\right\|_{K} \\
& \leq \Pi_{j=0}^{t} \frac{j+t_{0}-1}{j+t_{0}}\left\|f_{0}-f_{\rho}\right\|_{K} \\
& =\frac{t_{0}}{t+t_{0}}\left\|f_{\rho}\right\|_{K} .
\end{aligned}
$$


For the sample error, it is more difficult and the Pinelis-Bernstein inequality [4] will be applied.

Lemma 3 Let $\xi_{i}$ be a martingale difference sequence in a Hilbert space. Suppose that almost surely $\left\|\xi_{i}\right\| \leq B$ and $\sum_{i=1}^{t} \mathbb{E}_{i-1}\left\|\xi_{i}\right\|^{2} \leq \sigma_{t}^{2}$ for some constants $B, \sigma_{t}>0, t=1,2, \cdots$. Then for any $0<\delta<1$, with probability at least $1-\delta$, there holds

$$
\left\|\sum_{i=1}^{t} \xi_{i}\right\| \leq 2\left(\frac{B}{3}+\sigma_{t}\right) \ln \left(\frac{2}{\delta}\right)
$$

Now the error bounds for sample error can be derived. Notice that

$$
\begin{aligned}
& \left\|B_{t}\right\|_{K} \leq \eta_{t}\left[M \kappa+\left(\kappa^{2}+\lambda_{t}\right)\left\|f_{\rho}\right\|_{K}\right] \leq \eta_{t}\left[M \kappa+\left(\kappa^{2}+1\right)\left\|f_{\rho}\right\|_{K}\right] \text {. Set } \\
& \xi_{i}=A_{t} A_{t-1} \cdots A_{i} B_{i-1}, i=1,2, \cdots, t, \text { then } \\
& \left\|\xi_{i}\right\|_{K} \leq\left\|A_{t}\right\|\left\|A_{t-1}\right\| \cdots\left\|A_{i}\right\|\left\|B_{i-1}\right\|_{K} \leq \frac{i+t_{0}-1}{t+t_{0}} \eta_{i-1}\left[M \kappa+\left(\kappa^{2}+1\right)\left\|f_{\rho}\right\|_{K}\right] \\
& =\frac{1}{t+t_{0}} \frac{1}{\lambda_{i-1}}\left[M \kappa+\left(\kappa^{2}+1\right)\left\|f_{\rho}\right\|_{K}\right] \leq \frac{1}{t+t_{0}} \frac{1}{\lambda_{t}}\left[M \kappa+\left(\kappa^{2}+1\right)\left\|f_{\rho}\right\|_{K}\right] \\
& =\eta_{t}\left[M \kappa+\left(\kappa^{2}+1\right)\left\|f_{\rho}\right\|_{K}\right] .
\end{aligned}
$$

And $\sum_{i=1}^{t} \mathbb{E}_{i-1}\left\|X_{i}\right\|_{K}^{2} \leq t \eta_{t}^{2}\left[M \kappa+\left(\kappa^{2}+1\right)\left\|f_{\rho}\right\|_{K}\right]$. So for any $0<\delta<1$, with probability at least $1-\delta$,

$$
\begin{aligned}
\left\|\sum_{i=1}^{t} \xi_{i}\right\|_{K} & \leq \frac{8}{3} \sqrt{t} \eta_{t}\left[M \kappa+\left(\kappa^{2}+1\right)\left\|f_{\rho}\right\|_{K}\right] \ln \left(\frac{2}{\delta}\right) \\
& \leq \frac{8}{3 t^{\theta-1 / 2}}\left[M \kappa+\left(\kappa^{2}+1\right)\left\|f_{\rho}\right\|_{K}\right] \ln \left(\frac{2}{\delta}\right)
\end{aligned}
$$

Note that $\left\|B_{t}\right\|_{K} \leq \eta_{t}\left[M \kappa+\left(\kappa^{2}+1\right)\left\|f_{\rho}\right\|_{K}\right]$, hence

$$
\left\|B_{t}+A_{t} B_{t-1}+\cdots+A_{t} A_{t-1} \cdots A_{1} B_{0}\right\|_{K} \leq \frac{11}{3 t^{\theta-1 / 2}}\left[M \kappa+\left(\kappa^{2}+1\right)\left\|f_{\rho}\right\|_{K}\right] \ln \left(\frac{2}{\delta}\right)
$$

Combining the initial error, sample error bounds and applying Markov inequality for the fact that $\mathbb{E}\left|b_{t+1}\right|=C_{t+1} / \epsilon$, the total error estimation is obtained.

Theorem 2 Choose $\eta_{t}, \lambda_{t}$ and $b_{t}$ as in the theorem in the last section, with confidence $1-\delta(0<\delta<1)$, there holds

$$
\left\|f_{t+1, \mathcal{A}}-f_{\rho}\right\|_{K} \leq C_{\epsilon} \frac{1}{t^{\theta-1 / 2}} \frac{4}{\delta}
$$

where constant $C_{\epsilon}=4\left(\kappa^{2}+1\right) \kappa M / \epsilon+t_{0}\left\|f_{\rho}\right\|_{K}+\frac{11}{3}\left(\kappa M+\left(\kappa^{2}+1\right)\left\|f_{\rho}\right\|_{K}\right)$.

\section{Conclusion}

In this paper, analysis is performed for the differential privacy (Theorem 1) and generalization property (Theorem 2) for the online differential private learning algorithm (6). Under the choice of parameters in our theorems, the algorithm (6) can provide $\epsilon$-differential privacy and keep learning rate close to $1 / 2$, for any $\epsilon>0$. However, this error bound is not satisfactory enough. It might be an interesting problem to promote the error bound from $2 / \delta$ to $\ln (2 / \delta)$ in our 
future work.

\section{Fund}

This work is supported by NSFC (Nos. 11326096, 11401247), NSF of Guangdong Province in China (No. 2015A030313674), Foundation for Distinguished Young Talents in Higher Education of Guangdong, China (No. 2013LYM_0089), National Social Science Fund in China (No. 15BTJ024), Planning Fund Project of Humanities and Social Science Research in Chinese Ministry of Education (No. 14YJAZH040) and Doctor Grants of Huizhou University (No. C511.0206).

\section{References}

[1] Ying, Y. and Zhou, D.X. (2006) Online Regularized Classification Algorithms. IEEE Transactions on Information Theory, 11, 4775-4788. https://doi.org/10.1109/TIT.2006.883632

[2] Smale, S. and Zhou, D.X. (2009) Online Learning with Markov Sampling. Analysis and Applications, 7, 87-113. https://doi.org/10.1142/S0219530509001293

[3] Guo, Z.C. and Wang, C. (2011) Online Regression with Unbounded Sampling. International Journal of Computer Mathematics, 88, 2936-2941. https://doi.org/10.1080/00207160.2011.587510

[4] Tarrés, P. and Yao, Y. (2014) Online Learning as Stochastic Approximations of Regularization Paths. IEEE Transactions on Information Theory, 60, 5716-5735.

[5] Ying, Y. and Zhou, D.X. (2015) Online Pairwise Learning Algorithms with Kernels. Computer Science, 10, 441-449.

[6] Dwork, C., McSherry, F., Nissim, K. and Smith, A. (2006) Calibrating Noise to Sensitivity in Private Data Analysis. Theory of Cryptography, Springer, 265-284. https://doi.org/10.1007/11681878_14

[7] Jain, P., Kothari, P. and Thakurta, A. (2012) Differential Private Online Learning. JMLR: Workshop and Conference Proceedings, 2012, 1-34.

[8] Smale, S. and Yao, Y. (2006) Online Learning Algorithms. Foundations of Computational Mathematics, 6, 145-170. https://doi.org/10.1007/s10208-004-0160-Z

[9] Cucker, F. and Smale, S. (2002) On the Mathematical Foundations of Learning. Bulletin of the American Mathematical Society, 39, 1-49. https://doi.org/10.1090/S0273-0979-01-00923-5

[10] Aronszajn, N. (1950) Theory of Reproducing Kernels. Transactions of the American Mathematical Society, 68, 337-404. https://doi.org/10.1090/S0002-9947-1950-0051437-7

[11] Dwork, C. (2006) Differential Privacy. ICALP, Springer, 1-12. https://doi.org/10.1007/11787006_1 
Submit or recommend next manuscript to SCIRP and we will provide best service for you:

Accepting pre-submission inquiries through Email, Facebook, LinkedIn, Twitter, etc. A wide selection of journals (inclusive of 9 subjects, more than 200 journals)

Providing 24-hour high-quality service

User-friendly online submission system

Fair and swift peer-review system

Efficient typesetting and proofreading procedure

Display of the result of downloads and visits, as well as the number of cited articles Maximum dissemination of your research work

Submit your manuscript at: http://papersubmission.scirp.org/

Or contact jec@scirp.org 